\title{
Ticagrelor versus ASS bei Patienten nach Schlaganfall oder TIA
}

Fragestellung: Ist Ticagrelor wirksamer in der Sekundärprävention des Schlaganfalls als Acetylsalicylsäure (ASS)?

Hintergrund: Bei Patienten, bei denen nach einer transitorischen ischämischen Attacke (TIA) oder einem ischämischen Insult Vorhofflimmern ausgeschlossen ist, erfolgt die Sekundärprävention in der Regel mit ASS. In einer Metaanalyse zeigte sich eine $22 \%$ ige relative Risikoreduktion gegenüber Placebo oder keiner Therapie, wobei dieser Therapieeffekt relativ gering war. Ticagrelor ist ein Thrombozytenfunktionshemmer, der am P2Y12-Rezeptor von Thrombozyten reversibel bindet. Seine thrombozytenfunktionshemmende Wirkung ist im Gegensatz zu Clopidogrel unabhängig von genetischen Faktoren. In einer Sekundärpräventionsstudie bei Patienten mit akutem Koronarsyndrom war Ticagrelor signifikant wirksamer als Clopidogrel [1]. In der vorliegenden großen internationalen Studie sollte jetzt Ticagrelor im Vergleich zu Acetylsalicylsäure bei Patienten nach TIA und ischämischen Insult untersucht werden.

Patienten und Methodik: Es handelte sich um eine internationale doppelblinde, randomisierte Studie, in die in 33 Ländern 13.199 Patienten mit leichten ischämischen Insulten und

Johnston SC, Amarenco P, Albers GW et al; SOCRATES Steering Committee and Investigators. Ticagrelor versus aspirin in acute stroke or transient ischemic attack. N Engl J Med 2016; 375: $35-43$
Hochrisiko-TIA-Patienten aufgenommen wurden. TIAPatienten mussten auf der $\mathrm{ABCD}^{2}$-Schlaganfallrisikoskala einen Wert von $\geq 4$ aufweisen. Patienten, die eine Lyse oder Rekanalisa- tion erhielten, wurden ausgeschlossen. Die Randomisierung erfolgte innerhalb von 24 Stunden. Ticagrelor wurde in einer Loading-Dosis von $180 \mathrm{mg}$ am Tag 1 gegeben, gefolgt von zweimal 90 mg über 90 Tage. ASS wurde in einer Loading-Dosis von $300 \mathrm{mg}$ am ersten Tag gegeben, gefolgt von $100 \mathrm{mg}$ für die nächsten 90 Tage. Der primäre Endpunkt war die Zeit bis zum Auftreten von Schlaganfall, Herzinfarkt oder Tod innerhalb von 90 Tagen.

Ergebnisse: 6.589 Patienten wurden in die Ticagrelor-Gruppe und 6.610 in die ASS-Gruppe randomisiert. Die Patienten waren im Mittel 66 Jahre alt und $42 \%$ waren Frauen. Etwa ein Drittel der Patienten hatte zum Zeitpunkt der TIA oder des Schlaganfalls ASS eingenommen. Das qualifizierende Ereignis war in $27 \%$ der Fälle eine TIA und in $73 \%$ ein ischämischer Insult. Zwei Drittel der Patienten hatten einen Wert auf der NIHSS von $\leq 3$ zum Zeitpunkt der Randomisierung.

Der primäre Endpunkt trat bei 442 Patienten in der Ticagrelor-Gruppe auf (6,7\%) und bei 497 in der ASS-Gruppe (7,5\%). Dies entsprach einer Hazard Ratio (HR) von 0,89, die mit einem p-Wert von 0,07 nicht signifikant war. Die Ergebnisse für erneute ischämische Insulte waren $5,8 \%$ versus $6,7 \%$, entsprechend einer signifikanten HR von 0,87 ( $p=0,046)$. Für die übrigen vaskulären Endpunkte ergaben sich keine signifikanten Unterschiede. Schwerwiegende Blutungen traten bei 31 versus $38 \mathrm{~Pa}$ tienten auf, entsprechend $0,5 \%$ und $0,6 \%$.

Schlussfolgerungen: Ticagrelor bewirkt eine nicht signifikante Reduktion vaskulärer Endpunkte nach TIA und leichtem Schlaganfall, ohne das Blutungsrisiko zu erhöhen.

\section{- Kommentar von Hans-Christoph Diener, Essen}

\section{Off-Label-Alternative für Patienten, die ASS nicht vertragen}

Die SOCRATES-Studie ist leider bezogen auf den kombinierten Endpunkt Schlaganfall, Herzinfarkt und Tod ganz knapp negativ ausgegangen. Für den relevanten Endpunkt Rezidive von ischämischen Insulten ergab sich eine signifikante Überlegenheit von Ticagrelor. Entgegen den Erwartungen war das Risiko von schwerwiegenden Blutungen unter Ticagrelor sogar tendenziell geringer als unter Acetylsalicylsäure. Für den klinischen Alltag bedeutet dies, dass Ticagrelor zur Sekundärprävention des Schlaganfalls nicht zugelassen werden wird. Es wäre im Off-Label-Einsatz aber eventuell eine Alternative für Patienten die ASS wegen Magenbeschwerden nicht vertragen.

Referenz:

1. James SK et al. Circulation 2012; 125: $2914-21$

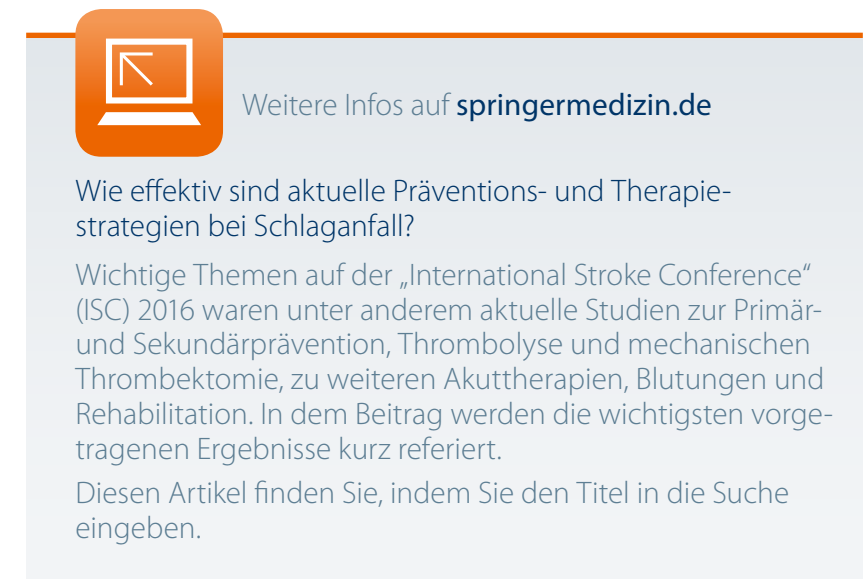

